\title{
Bundeskanzlerin Merkel zu Besuch bei Galvanotechnik Baum
}

leich drei Jubiläen wurden am 11.

3 März 2013 in Unterheinsdorf gefeiert: 35 Jahre Galvanotechnik Baum, 20 Jahre Galvanotechnik Baum in Zwönitz und 5 Jahre Galvanotechnische Oberflächen in Heinsdorfergrund. Zum Festakt „Innovation Zukunft 2013 - Tag der Technik 2020" mit insgesamt über 600 Gästen empfing das Unternehmen Bundeskanzlerin Dr. Angela Merkel als Ehrengast. Begleitet wurde sie vom sächsischen Ministerpräsidenten Stanislaw Tillich. Gemeinsam mit der Geschäftsführung um Nicolai A.J. Baum drückten sie den Startknopf für den Bau einer neuen, hoch leistungsfähigen Zink-Nickel-Gestellanlage, die bereits im September in Betrieb gehen soll. In der Gesamtinvestition von sieben Millionen Euro ist ein neues Kundenbetreuungszentrum eingeschlossen. Bundeskanzlerin Merkel lobte Galvanotechnik Baum in ihrer Rede für die Werte, die auch im Unternehmen gelebt werden. Beim Firmenrundgang informierte sie sich unter anderem über die Veredelung von Bremsenteilen für Bosch, die später bei VW und BMW zum Einsatz kommen.

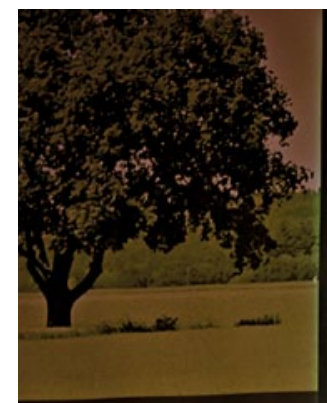

GREEN
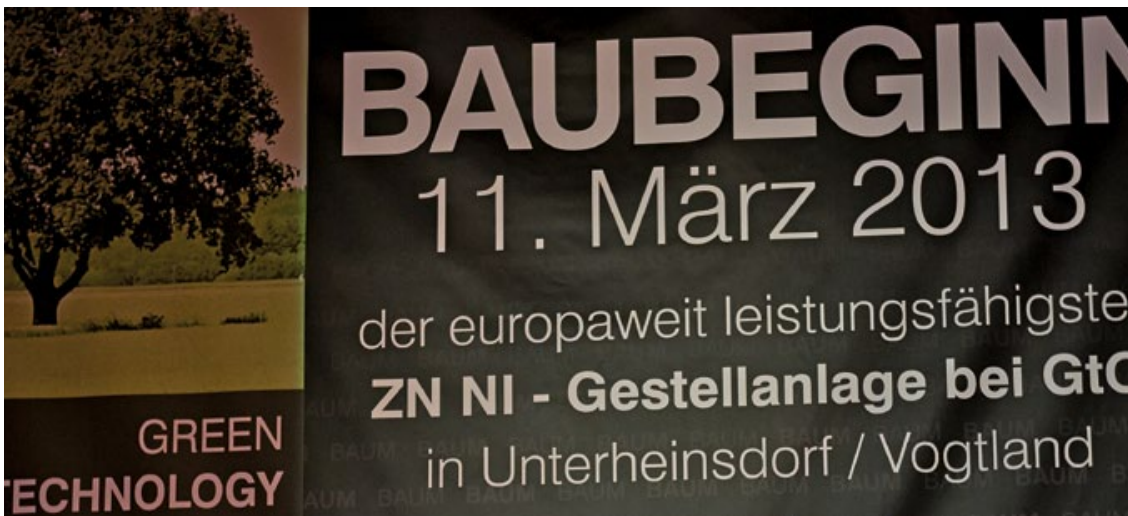

der europaweit leistungsfähigste ZN NI - Gestellanlage bei GtC in Unterheinsdorf / Vogtland

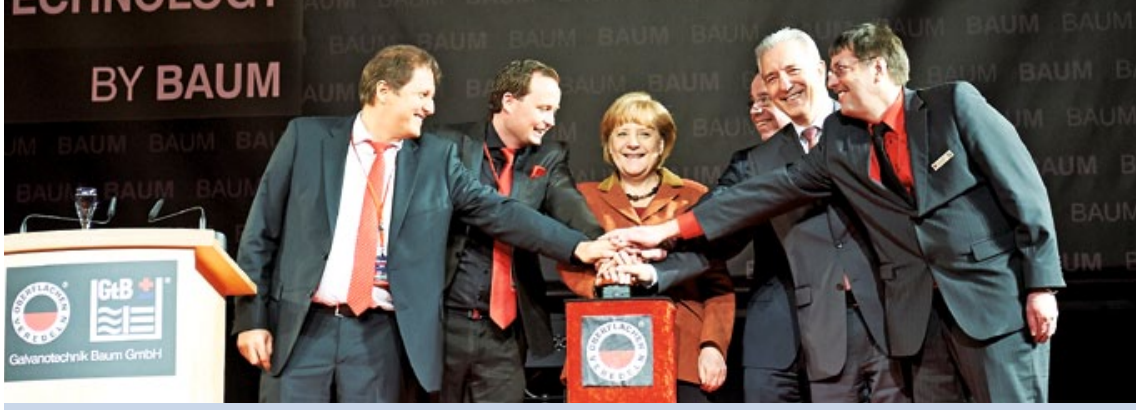

Offizieller Start zum Bau der Zink-Nickel-Gestellanlage (v.l.n.r.): Werner Wackershauser (Geschäftsführer Galvanotechnische Oberflächen), Nicolai A.J. Baum (Generalgeschäftsführung für Galvanotechnik Baum und Galvanotechnische Oberflächen), Bundeskanzlerin Angela Merkel, Oliver Jäger (Geschäftsführer Galvanotechnik Baum), Sachsens Ministerpräsident Stanislaw Tillich, Bernd Schwarzer (Technischer Geschäftsführer Galvanotechnik Baum)

\section{Tonnen Pulverlack für Warschaus neues Wahrzeichen}

Zsions ota ist ein Solitär in bester Innenstadtlage Warschaus, dessen auffällig gestaltete Fassade Blicke anzieht. Für den dauerhaften Schutz der 87000 Quadratmeter großen Fassade vor Wind und Wetter wurden 13 Tonnen Pulverlack verarbeitet. Entworfen wurde das 192 hohe Gebäude von Daniel Libeskind.
Der Stararchitekt betrachtet den Neubau als Symbol des politischen Wandels in Polens Hauptstadt. Charakteristisches Merkmal des Entwurfes ist die asymmetrisch geschwungene, aus verschiedenen Geometrien kunstvoll zusammengesetzte Silhouette. Diese dynamische Formgebung des Hochhau-

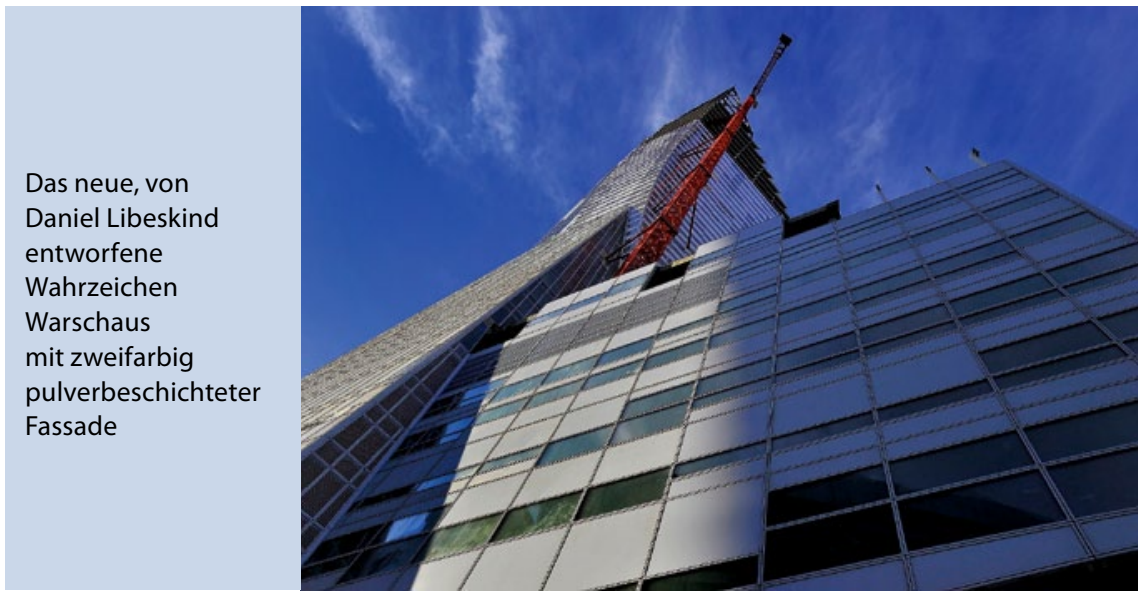

ses, durch die es bei den Warschauern auch den Beinamen "Segel" erhielt, erforderte ein entsprechendes Fassadensystem. Die Einzelelemente dafür wurden in Form und Abmessung für viele Bereiche des Gebäudes speziell angefertigt. Aufgrund des hohen Aufwandes bei der Fassadengestaltung und des repräsentativen, aufmerksamkeitsstarken Bauwerks, spielte ein stabiler, qualitativ hochwertiger Vorbehandlungs- und Beschichtungsprozess eine wichtige Rolle. Hier fiel die Entscheidung auf den belgischen Lohnbeschichter Blyweert und auf Pulverlacke (Alesta AP RAL 9010 matt smooth und NCS S0515 R80 B fine textured) von DuPont CoatingSolutions. Nach einigen Turbulenzen, verursacht durch die Finanzkrise und baurechtliche Herausforderungen, die den Bau für eine gewisse Zeit ins Stocken brachten, soll "Zlota“ 2013 endgültig fertiggestellt werden. 